\title{
Chemical enrichment in the early Galaxy
}

\author{
Torgny Karlsson ${ }^{1,2}$ \\ ${ }^{1}$ NORDITA, AlbaNova University Center, SE-106 91, Stockholm, Sweden \\ ${ }^{2}$ School of Physics, The University of Sydney, 2006 NSW, Australia \\ email: torgny@physics.usyd.edu.au
}

\begin{abstract}
The chemical enrichment by the first sources of light in the universe ultimately set the stage for the subsequent evolution of the Milky Way system. The oldest and, usually, the most-metal poor stars are our 'near-field' link to this ancient epoch as they, apart from tracing the chemical enrichment itself, also indirectly hold information on, e.g., the conditions for star formation and feed-back effects in the early universe. In particular, I will discuss the possible origins of the relatively large number of carbon enhanced metal-poor stars in the Galactic halo. Furthermore, I will argue that the apparent absence of the chemical signature of so-called pairinstability supernovae (PISNe), which are a natural consequence of current theoretical models for primordial star formation at the highest masses, may arise from a subtle observational selection effect. Whereas most surveys traditionally focus on the most metal-poor stars, early PISN enrichment is predicted to 'overshoot', reaching enrichment levels of $[\mathrm{Ca} / \mathrm{H}] \sim-2.5$ that would be missed by current searches.
\end{abstract}

Keywords. stars: abundances, stars: formation, stars: Population II, Galaxy: evolution, Galaxy: formation, Galaxy: halo

\section{Introduction}

Extremely metal-poor (EMP) stars are defined to have $[\mathrm{Fe} / \mathrm{H}]<-3 \dagger$ (Beers \& Christlieb 2005) and they populate the metal-poor tail of the Galactic halo metallicity distribution function (MDF). Curiously, EMP stars appear to be the only objects in the known universe found to have metallicities significantly below $[\mathrm{Fe} / \mathrm{H}]=-3$ and as such, they are commonly believed to be, possibly, our closest link (i.e., both spatially and relation-wise) to the era of the first stars.

There is an ongoing debate on which types of systems that were the original building blocks of the Galactic halo. In accordance with the $\Lambda$ CDM paradigm, it has been argued that systems similar to those of the dwarf spheroidal (dSph) satellite galaxies were a dominant formation site of Galactic halo stars. However, this view appear to be incompatible with the low levels of $\alpha$-enhancement observed in individual dSph stars (e.g., Venn et al. 2004) and rules out present-day dSphs, like Fornax and Sculptor, as the formation site of Galactic halo stars in general, at least the high- $\alpha$ stars (see contribution by P. E. Nissen, this volume). It also turns out that the observed lack of EMP stars in the more luminous dSphs (Helmi et al. 2006) appear to rule out these objects as the formation site of the Galactic halo EMP stars in particular. These dSphs probably formed relatively late, in lower-density peaks already enriched to a level of $[\mathrm{Fe} / \mathrm{H}] \simeq-3$ (Helmi et al. 2006; Salvadori et al. 2008).

At present, it is not clear exactly in which environment the EMP stars were formed originally. Since they are believed to be second generation stars, born out of gas enriched

$\dagger[\mathrm{A} / \mathrm{B}]=\log _{10}\left(N_{A} / N_{B}\right)_{\star}-\log _{10}\left(N_{A} / N_{B}\right) \odot$, where $N_{X}$ is the number density of element $X$. 
only by the first supernovae ( $\mathrm{SNe}$ ), the majority of these stars must have formed relatively early on, presumably in so-called 'atomic cooling halos', which are small dark matter halos on the order of $10^{8} \mathcal{M}_{\odot}$ (see, e.g., Greif et al. 2008). These halos were probably the first objects able to form Pop II stars (e.g., Karlsson et al. 2008). Interestingly, the newly discovered ultra-faint dSphs (e.g., Willman et al. 2005; Belokurov et al. 2007), which may be relic galaxies formed prior to reionization (Bovill \& Ricotti 2008), appear to contain a fair fraction of EMP stars (Kirby et al. 2008). If so, these objects may turn out to be surviving members of a population of 'first galaxies' that would give us a unique possibility to directly study the birth sites of second generation EMP stars.

\section{Star-to-star scatters in chemical abundance ratios for EMP stars}

Recent observations by a number of authors (e.g., Cayrel et al. 2004; Arnone et al. 2005; Barklem et al. 2005) have revealed surprisingly small star-to-star scatters in the $\alpha$ and iron-peak elements for very metal-poor and EMP stars, scatters which are consistent with observational uncertainties alone. This has been interpreted as evidence of a chemically well-mixed interstellar medium (ISM), even at very early epochs, in which the mixing time-scale was extremely short.

To circumvent unphysically short mixing time-scales, Arnone et al. (2005) speculated that the longer cooling time-scales in metal-poor gas may prevent subsequent star formation until the ejecta from whole generations of SNe were efficiently mixed. As a result, a small star-to-star scatter would be achieved. However, very long cooling time-scales would wipe out any observed trend with metallicity, such as a decreasing [Zn/Fe] with increasing $[\mathrm{Fe} / \mathrm{H}]$ observed for EMP giants (Cayrel et al. 2004), unless the SN yields, integrated over the initial mass function (IMF), show a significant metallicity dependence. At least in the case of $[\mathrm{Zn} / \mathrm{Fe}]$, theoretical yield calculations do not seem to predict such a dependence (e.g., Chieffi \& Limongi 2004; Nomoto et al. 2006). Another interesting example is the decreasing $[\mathrm{C} / \mathrm{O}]$ trend with increasing $[\mathrm{O} / \mathrm{H}]$ observed for EMP stars (Akerman et al. 2004). Also worth noting is that, even though all stars may form in clusters, at least in the Galaxy today, the majority $(\sim 50-85 \%)$ of the massive stars exploding as $\mathrm{SNe}$, will do so in isolation and not in groups. Hence, the extra averaging of SN ejecta due to clustered star formation will probably not be very significant.

In fact, the presence of trends in the EMP Galactic halo may instead suggest that the ISM at this point was fairly unmixed. In a poorly mixed, extremely metal-poor ISM, two low-mass stars can form out of gas enriched by two SNe of different masses producing different amounts of heavy elements. In such a scenario, trends may simply result from the different mass-dependences of the SN yields (Karlsson \& Gustafsson 2005; Nomoto et al. 2006). However, in contrast to what is assumed in homogeneous chemical evolution models, where the most metal-poor stars are enriched by the stars with the shortest lifetimes, i.e., the highest-mass stars, the most metal-poor stars will instead, in most cases, be enriched by the least massive SNe as they eject the least amount of metals.

A number of other elements instead show clear evidence of inhomogeneous chemical enrichment. Large and real scatters are found in the n-capture elements (e.g., François et al. 2007) and probably also in elements like $\mathrm{Na}, \mathrm{Al}$, and $\mathrm{Si}$ (Cayrel et al. 2004) as well as in N (Spite et al. 2005). Although the scatter in the n-capture elements may result from the possibility that these elements only are produced in a small SN mass range as compared to light and intermediate-mass elements (e.g., Cescutti 2008), this would not explain the observed scatter in those other elements. As regards nitrogen, Chiappini et al. (2006) speculate that the large observed scatter may originate from variations in 
the initial rotational velocity of extremely metal-poor massive stars, which are shown to produce large variations in the N-yield (Hirschi 2007).

The issue of the star-to-star scatter dichotomy, where some elements show evidence of an unmixed ISM while others do not, needs to be further addressed. However, worth keeping in mind is that 1) incomplete and/or biased samples of stars may not capture the true cosmic star-to-star scatter, I will briefly come back to this in Sect. 5, and 2) the effects of inhomogeneous chemical enrichment are only expected to be observed below $[\mathrm{Fe} / \mathrm{H}] \sim-3$ (Karlsson 2005), a metallicity regime which still is quite poorly sampled.

\section{Carbon-enhanced metal-poor stars}

One of the major discoveries found by the HK and the Hamburg/ESO surveys of metalpoor stars is the large fraction of metal-poor stars having $[\mathrm{C} / \mathrm{Fe}]>1$. These stars are called carbon-enhanced, metal-poor (CEMP) stars. The fraction of CEMP stars below $[\mathrm{Fe} / \mathrm{H}]=-2$ is determined to $\sim 10-20 \%$ (e.g., Cohen et al. 2005; Lucatello et al. 2006), a fraction which tend to increase with decreasing metallicity. About $80 \%$ of the CEMP stars have been shown to be enhanced in s-process elements as well. This subgroup is named CEMP-s, and there are now convincing evidence that the vast majority of the CEMP-s stars are members of binary systems and that they, like the more metal-rich $\mathrm{CH}$ and Ba stars, likely obtained their peculiar abundance pattern by mass-transfer from an evolved primary (e.g., Lucatello et al. 2005).

What about the remaining $20 \%$ of the CEMP stars, the so-called CEMP-no stars, "no" standing for no s-process enrichment? Here, the CEMP-r stars, which are enhanced in r-process (but no s-process) elements, are included in this group. Interestingly, the three ultra metal-poor stars (see Beers \& Christlieb 2005) found below $[\mathrm{Fe} / \mathrm{H}]=-4.5$, HE 0107 - 5240 (Christlieb et al. 2002), HE 1327 - 2326 (Frebel et al. 2005), and HE $0557-4840$ (Norris et al. 2007) are all CEMP-no stars and none of them has a detected binary companion. A number of different scenarios have been put forward to explain the anomalous abundance patterns, in particular the high CNO abundances, observed in these ultra metal-poor stars. These scenarios include pre-enrichment of the primordial cloud by a faint SN (e.g., Umeda \& Nomoto 2003), enrichment by massive star winds (Meynet et al. 2006), mass-transfer from a binary companion (e.g., Suda et al. 2004; Tumlinson 2007), and atmospheric dust-gas separation (Venn \& Lambert 2008).

Instead of treating the ultra metal-poor stars individually, as unique objects, it could, alternatively, be worthwhile to explore what one may learn if they were treated as 'normal' stars in the general context of chemical evolution. If the gas out of which the EMP stars were formed, experienced a period of low or delayed star formation, e.g., due to negative feedback effects from the first generations of stars, a small population of very C-enhanced, ultra metal-poor stars is to expect (Karlsson 2006). Figure 1 shows the predicted distribution of EMP stars (shaded areas) in the $[\mathrm{C} / \mathrm{Fe}]-[\mathrm{Fe} / \mathrm{H}]$ plane. Evidently, the predicted fraction of CEMP stars increases with decreasing $[\mathrm{Fe} / \mathrm{H}]$, which partly is due to the fact that low-mass star formation may be inhibited in regions with low contents of C (and O, see, e.g., Frebel et al. 2007). The predicted fraction of CEMP-no stars below $[\mathrm{Fe} / \mathrm{H}]=-2$ is roughly $1-7 \%$, depending on which set of stellar yields is used. This is consistent with the observed fraction of $2-5 \%$.

As a result of the delayed star formation, the current model is also able to explain the apparent deficit of stars in the metallicity range $-5 \lesssim[\mathrm{Fe} / \mathrm{H}] \lesssim-4$. Note that the shortfall of stars in this region, should, in this scenario, rather be interpreted as an extension or a stretching of the Galactic halo $\mathrm{MDF}$ below $[\mathrm{Fe} / \mathrm{H}] \simeq-4$. Finally, a small 


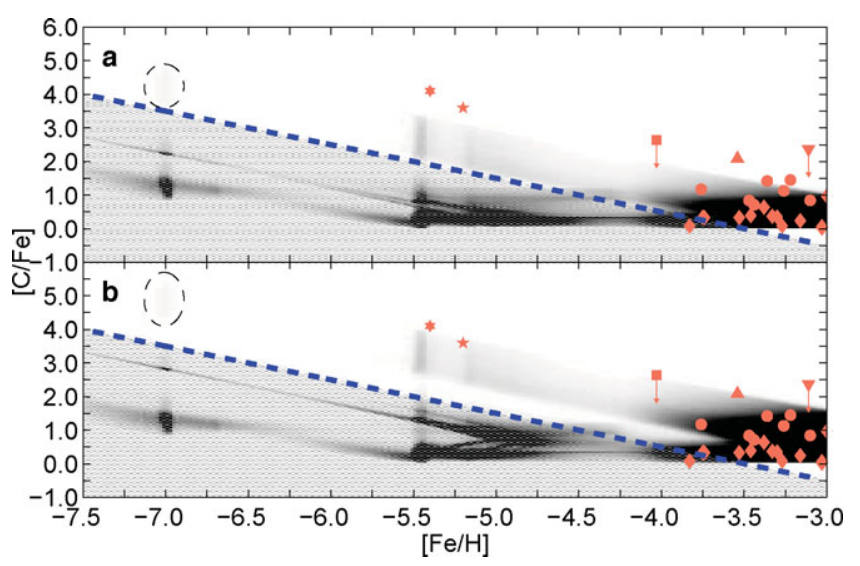

Figure 1. The predicted distribution of stars in the $[\mathrm{C} / \mathrm{Fe}]-[\mathrm{Fe} / \mathrm{H}]$ plane. Upper panel: The thick, dashed line indicates a carbon abundance of $[\mathrm{C} / \mathrm{H}]=-3.5$. The shaded area below this limit should contain very few low-mass stars. Symbols denote various observations of stars in the Milky way's halo (see Karlsson 2006). The predicted group of mega metal-poor stars is encircled. Lower panel: Same as above but with a carbon yield increased by a factor of 4 for stars in the mass range $30 \leqslant m / \mathcal{M}_{\odot} \leqslant 60$. Observed abundance ratios are not corrected for $3 \mathrm{D}$ effects.

population of mega metal-poor stars $([\mathrm{Fe} / \mathrm{H}]<-6)$ is predicted to exist in this scenario as a result of an early enrichment by O-Ne-Mg SNe (see Figure 1; Karlsson 2006).

\section{The missing second stellar generation}

Current simulations of primordial star formation tend to agree on the fact that the initial conditions in the early universe should favor the formation of massive and verymassive stars, in excess of $100 \mathcal{M}_{\odot}$ (e.g., Bromm et al. 1999,2002; Abel et al. 2002). This is partly due to the inefficient cooling of the gas which result in initially high Jeans masses (e.g., Larson \& Starrfield 1971) and partly due to the apparent inability of primordial collapsing gas clouds to fragment at later stages (Yoshida et al. 2006). Interestingly, Eta Carinae and the Pistol Star are two examples of very massive stars in our own galaxy and recently, an extremely powerful SN explosion in the nearby galaxy NGC 1260 was suggested to be the final death of a very massive star in excess of $100 \mathcal{M}_{\odot}$ (Smith et al. 2007).

Assuming that primordial very massive stars were able to form and that at least some of them where able to explode as so-called pair-instability SNe (PISNe), in what metallicity regime should we expect to find their chemical signature? Due to their very short life-times, PISNe must have been the first stars to enrich the ISM in metals. In the classical picture of chemical evolution, an initial enrichment by primordial PISNe would generate a metallicity floor out of which the second generation, low-mass stars were able to from. Naturally, these stars would be the most metal-poor stars to be found in the Galaxy and would show a unique PISN signature, characterized by, by not limited to, a pronounced odd-even effect and a lack of $\mathrm{r}$ - and s-process elements. Such a signature is, however, not observed, neither in the EMP stars, nor in the ultra metal-poor stars. These stars all show chemical signatures more resembling those of normal core collapse SNe (see, however, Sect. 3 for a further discussion). This has been taken as an indication that very massive stars were exceedingly few in the early universe (e.g., Tumlinson et al. 2004; Ballero et al. 2006), if not altogether absent, in contrast to the predictions.

A rather different conclusion may, however, be drawn if the instantaneous mixing approximation assumed in homogeneous chemical evolution modelling is relaxed and 


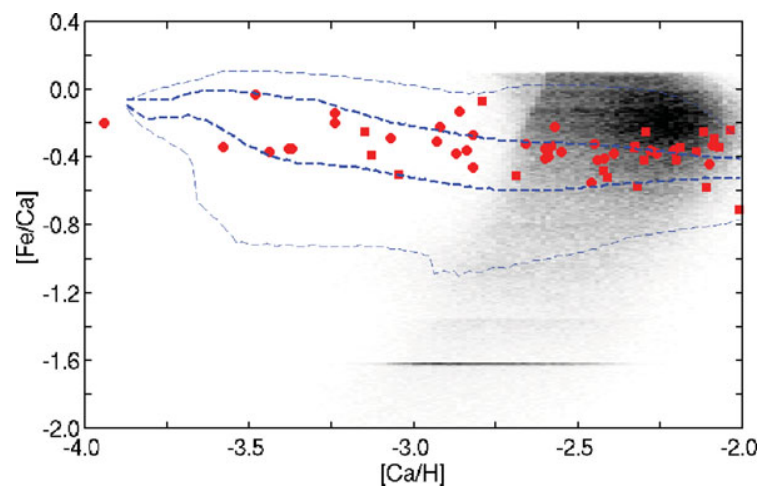

Figure 2. Predicted distribution (shaded area) of low-mass stars in the $[\mathrm{Fe} / \mathrm{Ca}]-[\mathrm{Ca} / \mathrm{H}] \mathrm{plane}$. Only the partial distribution of simulated stars for which $>90 \%$ of the total atmospheric Ca abundance is synthesized in PISNe is displayed. Symbols denote various observations of Galactic halo stars. The dashed, thick and thin lines indicate, respectively, the $1 \sigma$ (innermost $68.3 \%$ ) and $3 \sigma$ (innermost $99.7 \%$ ) cosmic scatter in the simulation (for details, see Karlsson et al. 2008).

the enrichment by SNe is instead allowed to occur locally, where the ejecta are mixed relatively slowly with their surroundings by turbulent diffusion. In order to simulate the stochastic enrichment by primordial PISNe in a cosmological context, we followed the initial star formation and chemical enrichment of collapsing atomic cooling halos (Karlsson et al. 2008). We allowed for a primordial star formation mode in which only massive and very massive stars were able to form. This primordial mode ceased as the gas became metal-enriched and turned into a normal Pop II star formation mode in which only low- and high mass stars were allowed to form. The larger explosion energies of the PISNe was also taken into account as they may sweep up larger amounts of gas before merging with the ISM.

The result is shown in Figure 2. The predicted distribution of stars with a dominant contribution from primordial PISNe, here shaded in gray, is located around $[\mathrm{Ca} / \mathrm{H}] \sim$ -2.5 , significantly above the most metal-poor stars in the simulation (indicated by the thick and thin dashed lines). These stars have, in fact, such high Ca abundances that a fair fraction of them may risk to remain undetected in current surveys of metal-poor stars. Furthermore, for realistic estimates of the fraction of primordial very massive stars exploding as PISNe (say $\sim 10 \%$ by mass in the primordial stellar population, Greif \& Bromm 2006), the fraction of low-mass stars below $[\mathrm{Ca} / \mathrm{H}]=-2$ with a dominant PISN signature is predicted to be very low, only $\sim 3 \times 10^{-4}$, which may well explain the fact that such a star has not yet been found. Current observational data are able to constrain the fraction of metal-free, very massive stars to $\lesssim 40 \%$, by mass (Karlsson et al. 2008).

\section{Concluding remarks}

Although the EMP stars are crucial for the understanding of the star formation process, feedback effects and the overall chemical enrichment in the early Galaxy, they do seem to tell us little about the primordial PISNe. Instead, it may be the "not-so-extremelymetal-poor" stars that hold the clue to the first sources of light in the universe, i.e., if our understanding of primordial star formation is correct. Furthermore, as argued in Sect. 3, CEMP-no stars belong to a population of stars which was born with a high $[\mathrm{C} / \mathrm{Fe}]$. If so, these stars should not be excluded from the general picture of chemical evolution. In particular, they should be considered when computing the cosmic star-to-star scatter. 
Acknowledgment. The author acknowledges support from the IAU and Danish sponsors.

\section{References}

Abel, T., Bryan, G. L., \& Norman, M. L. 2002, Science, 295, 93

Akerman, C. J., Carigi, L., Nissen, P. E., Pettini, M., \& Asplund, M. 2004, A\& A, 414, 931

Arnone, E., Ryan, S. G., Argast, D., Norris, J. E., \& Beers, T. C. 2005, A\&A, 430, 507

Ballero, S. K., Matteucci, F., \& Chiappini, C. 2006, New Astron., 11, 306

Barklem, P. S., et al. 2005, A\&SA, 439, 129

Beers, T. C. \& Christlieb, N. 2005, ARAA, 43, 531

Belokurov, V., et al. 2007, ApJ, 654, 897

Bovill, M. S. \& Ricotti, M. 2008, ApJ, submitted (astro-ph/0806.2340)

Bromm, V., Coppi, P. S., \& Larson, R. B. 1999, ApJ (Letters), 527, L5

-. 2002, ApJ, 564, 23

Cayrel, R., et al. 2004, A\&A, 416, 1117

Cescutti, G. 2008, $A \& A$, 481, 691

Chiappini, C., Hirschi, R., Meynet, G., Ekström, S., Maeder, A., \& Matteucci, F. 2006, A\&A (Letters), 449, L27

Chieffi, A. \& Limongi, M. 2004, ApJ, 608, 405

Christlieb, N., et al. 2002, Nature, 419, 904

Cohen, J. G., et al. 2005, ApJ (Letters), 633, L109

François, P., et al. 2007, A\&̊A, 476, 935

Frebel, A., Johnson, J. L., \& Bromm, V. 2007, MNRAS (Letters), 380, L40

Frebel, A., et al. 2005, Nature, 434, 871

Greif, T. H. \& Bromm, V. 2006, MNRAS, 373, 128

Greif, T. H., Johnson, J. L., Klessen, R. S., \& Bromm, V. 2008, MNRAS, submitted (astro$\mathrm{ph} / 0803.2237)$

Helmi, A., et al. 2006, ApJ (Letters), 651, L121

Hirschi, R. 2007, $A \mathscr{E} A, 461,571$

Karlsson, T. 2005, A\&\&A, 439, 93

-. 2006, ApJ (Letters), 641, L41

Karlsson, T. \& Gustafsson, B. 2005, A\& $A$ A, 436, 879

Karlsson, T., Johnson, J. L., \& Bromm, V. 2008, ApJ, 679, 6

Kirby, E. N., Simon, J. D., Geha, M., Guhathakurta, P., \& Frebel, A. 2008, ApJ (Letters), submitted (astro-ph/0807.1925)

Larson, R. B. \& Starrfield, S. 1971, A\&A, 13, 190

Lucatello, S., Beers, T. C., Christlieb, N., Barklem, P. S., Rossi, S., Marsteller, B., Sivarani, T., \& Lee, Y. S. 2006, ApJ (Letters), 652, L37

Lucatello, S., Tsangarides, S., Beers, T. C., Carretta, E., Gratton, R. G., \& Ryan, S. G. 2005, ApJ, 625, 825

Meynet, G., Ekström, S., \& Maeder, A. 2006, A\&A, 447, 623

Nomoto, K., Tominaga, N., Umeda, H., Kobayashi, C., \& Maeda, K. 2006, Nucl. Phys. A, 777, 424

Norris, J. E., et al., N. 2007, ApJ, 670, 774

Salvadori, S., Ferrara, A., \& Schneider, R. 2008, MNRAS, 386, 348

Smith, N., et al. 2007, ApJ, 666, 1116

Spite, M., et al. 2005, A\&\&A, 430, 655

Suda, T., Aikawa, M., Machida, M. N., Fujimoto, M. Y., \& Iben, I., Jr. 2004, ApJ, 611, 476

Tumlinson, J. 2007, ApJ, 665, 1361

Tumlinson, J., Venkatesan, A., \& Shull, J. M. 2004, ApJ, 612, 602

Umeda, H. \& Nomoto, K. 2003, Nature, 422, 871

Venn, K. A., Irwin, M., Shetrone, M. D., Tout, C. A., Hill, V., \& Tolstoy, E. 2004, AJ, 128, 1177

Venn, K. A. \& Lambert, D. L. 2008, ApJ, 677, 572

Willman, B., et al. 2005, AJ, 129, 2692

Yoshida, N., Omukai, K., Hernquist, L., \& Abel, T. 2006, ApJ, 652, 6 